\title{
Nanocrystallites in tetrahedral amorphous carbon films
}

\author{
S. Ravi P. Silva ${ }^{a)}$ \\ Department of Electronics and Electrical Engineering, University of Surrey, \\ Guildford, Surrey GU2 5XH, United Kingdom
}

Shi Xu, B. X. Tay, and H. S. Tan

Nanyang Technology University, Singapore 639798, Singapore

W. I. Milne

Engineering Department, Cambridge University, Trumpington Street, Cambridge CB2 1PZ, United Kingdom

(Received 4 March 1996; accepted for publication 17 May 1996)

\begin{abstract}
The microstructure of filtered cathodic vacuum arc deposited tetrahedral amorphous carbon films is studied as a function of ion energy. An optimum energy window in the density and $\mathrm{C}-\mathrm{C} s p^{3}$ content at an ion energy of $\sim 90 \mathrm{eV}$ observed in this study. It is shown that the density of the amorphous carbon films are closely related to the $s p^{3}$ content. The observation of nanocrystals embedded in the amorphous carbon matrix is reported. Most of the crystals observed by transmission electron microscopy can be indexed to graphite, but some of the crystals can be indexed to cubic diamond. The chemical composition of the crystals is analyzed using electron energy loss spectroscopy (EELS). The only discernible EELS edge is that of $\mathrm{C}$ at an energy of 285 eV. (C) 1996 American Institute of Physics. [S0003-6951(96)03230-5]
\end{abstract}

Filtered cathodic vacuum arc (FCVA) deposited carbon films have the unique property of producing high quality diamondlike amorphous carbon films with a high $s p^{3}$ content. $^{1,2}$ The film properties are critically dependent on the ion energy of the species. The carbon plasma initiated from a graphite target by the vacuum arc is sustained during the deposition and macroparticles associated with the arc process are filtered using a $90^{\circ}$ bend toroidal solenoid in the deposition system. We have investigated the relationship between the bonding structure in the films and the ion energy of the $\mathrm{C}$ species. At a particular threshold ion energy the particles found in the tetrahedral amorphous carbon (ta-C) films have been studied using transmission electron microscopy (TEM) and electron energy loss spectroscopy (EELS). Although there are reports that diamond nanocrystallites have been observed in diamondlike carbon (DLC) films deposited under low temperature and low pressure conditions, ${ }^{3-6}$ direct observation of diamond crystals in deposition techniques that do not operate at high temperatures and high pressures is not common. This is because graphite is the stable crystalline form of carbon under these conditions.

Highly tetrahedrally bonded ta-C films have also been deposited using laser initiated vacuum arcs, and pulsed cathodic arcs, ${ }^{7,8}$ and hydrogenated ta-C films deposited using a plasma beam source. ${ }^{9}$ There is much interest in all of these films due to their unique mechanical properties. ${ }^{7,10}$ Recently, Yin et al. ${ }^{11}$ have found cauliflower-like growths (nanocrystals) which cannot be indexed to graphite in their FCVA deposited films, which are considered to be precursors for diamond growth. ${ }^{12}$ However, there has been no published literature on the observation of diamond nanocrystallites in ta-C films deposited using the FCVA. In this letter, we report on the observation of nanocrystallites that index to diamond,

${ }^{\text {a)} E l e c t r o n i c ~ m a i l: ~ s . s i l v a @ e e . s u r r e y . a c . u k ~}$ deposited under low temperature/low pressure conditions using such a system.

The FCVA films were deposited with a base pressure of $2 \times 10^{-6}$ Torr which increased to $1 \times 10^{-5}$ Torr during deposition. The substrate bias is varied from 0 to $-120 \mathrm{~V}$, while the arc current remained constant at $\sim 60 \mathrm{~A}$. A toroidal magnetic field of $40 \mathrm{mT}$ was employed to produce the axial and curvilinear fields. The $\mathrm{C}$ ions leave the self-sustaining arc, which is struck between the anode and compressed graphite target, with an average energy centered at $28 \mathrm{eV}$. A Philips CM30 TEM operated at $100 \mathrm{keV}$ was used together with a Gatan 666 parallel EELS system to analyze the microstructure of the films. A collection angle of 12 mrads was used to collect the EELS spectra. The $s p^{3}$ content was calculated using the method proposed by Berger et al. ${ }^{13}$ by analyzing the $\mathrm{C} K$ edge EELS spectrum. The low loss plasmon peak gives the valence electron density which may be used to determine the mass density of the ta-C films. ${ }^{14}$ The density measured is normalized to that of graphite.

The variation of the $s p^{3}$ fraction and the film density as a function of ion energy is shown in Fig. 1(a). A peak in both the $s p^{3}$ fraction as well as the density is observed at an ion energy of $88 \mathrm{eV}$. Peaks in the density versus ion energy curve seem to be system dependent for different research groups and have been observed at; 40, 92, 140, and a threshold value of $50 \mathrm{eV}$ in the results of McKenzie, ${ }^{15}$ Weiler, ${ }^{9}$ Fallon, ${ }^{2}$ and Lifshitz, ${ }^{16}$ respectively. The films deposited in the current work $^{17}$ use a similar apparatus to those used by McKenzie $^{15}$ and Fallon ${ }^{2}$. The applied magnetic fields influence the optimum energy window that controls the energy at which the peak in density occurs. At higher magnetic fields more doubly charged $\mathrm{C}^{2+}$ ions will be generated, ${ }^{18}$ and thereby a larger "thermal spike" relaxation process will result as these ions will have more energy to dissipate before they come to rest. The doubly charged carbon ions will move the peak in the density and $s p^{3}$ content to lower ion energy 

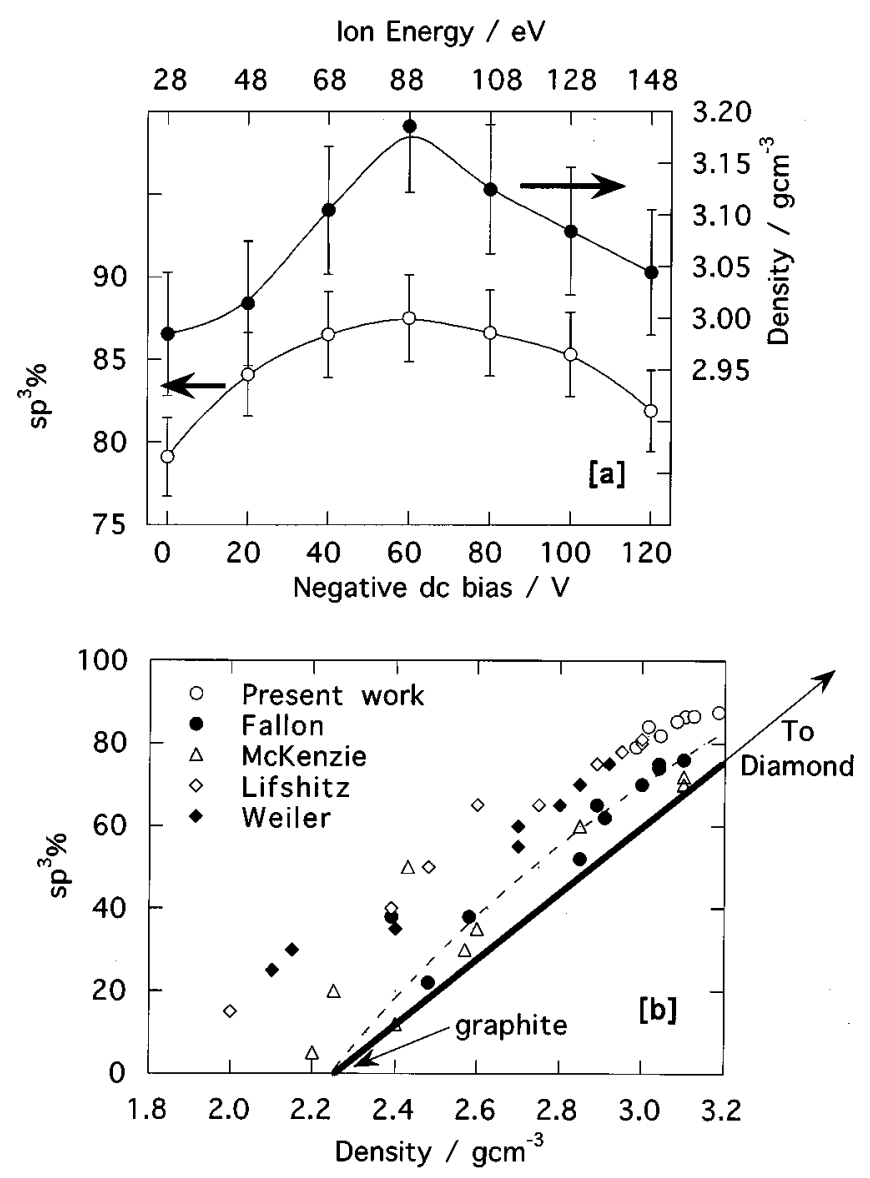

FIG. 1. (a) The variation of the $\mathrm{C} s p^{3}$ bonding and density in tetrahedral amorphous carbon films as a function of ion energy and negative dc bias voltage. (b) A comparison of the $s p^{3}$ bonding in tetrahedral amorphous carbon films as a function of the film density (Refs. 2, 9, 15, and 16). The variation predicted by Cuomo et al. (Ref. 21) using a "rule of mixtures" is shown by the dashed line.

values. The ta-C films deposited in this study used a magnetic field of $40 \mathrm{mT}\left(s p^{3}\right.$ peak at $\left.88 \mathrm{eV}\right)$, whereas films deposited $^{19}$ in an identical FCVA system with magnetic fields of 12 and $24 \mathrm{mT}$ gave $\mathrm{C} s p^{3}$ distributions which peaked at 130 and $90 \mathrm{eV}$, respectively. The predicted shift in the peak in the $s p^{3}$ to lower values is therefore observed. Recent results suggest that a higher ion energy due to the higher magnetic fields which modifies the plasma potential may have a more significant effect than the mean charge state of ions in the plasma. ${ }^{20}$ The observed $s p^{3}$ fraction of $\sim 87 \%$ at a density of $\sim 3.2 \mathrm{~g} \mathrm{~cm}^{-3}$ is comparable to the best results reported for ta-C films.

The peak in the $s p^{3}$ fraction and density occurring at the same ion energy is expected. The variation of the $s p^{3}$ fraction as a function of density for a number of research groups is shown in Fig. 1(b). Assuming that no $s p^{1}$ states and unsaturated bonds exist, the density of a nonhydrogenated carbon film must lie between those of graphite $\left(2.25 \mathrm{~g} \mathrm{~cm}^{-3}\right)$ and diamond $\left(3.5 \mathrm{~g} \mathrm{~cm}^{-3}\right)$. Yet, as shown in Fig. 1(b) the data for all the research groups lie above the line joining that of diamond and graphite. Cuomo et al. ${ }^{21}$ used a "rule of mixtures" to give a relationship between the $s p^{2} / s p^{3}$ bonds and density shown by the dashed line in the figure. Again, the fit to the data is not perfect and an overestimation of the density is predicted. The creation of a certain fraction of
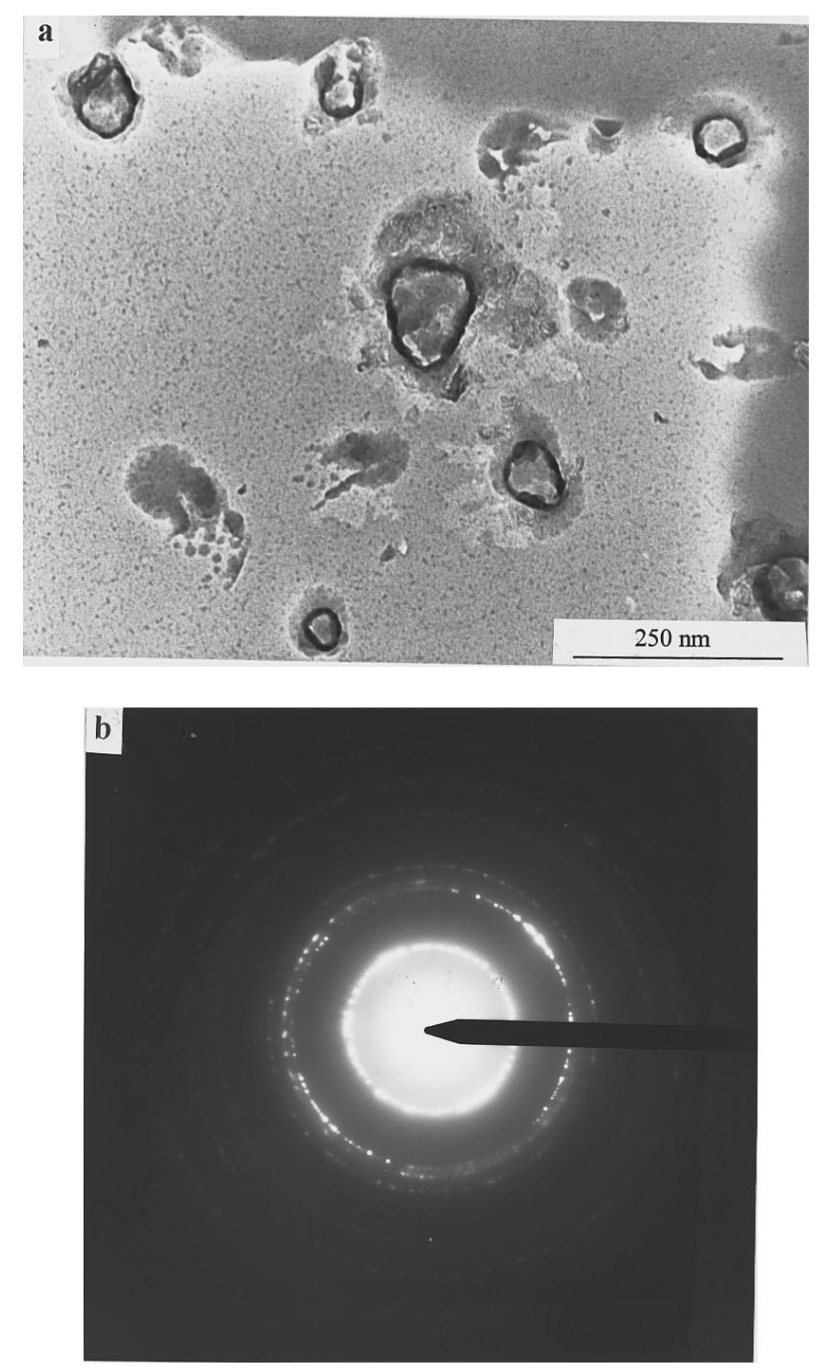

FIG. 2. (a) Bright field electron micrograph of the nanocrystallites that can be indexed to diamond and (b) the electron diffraction obtained from such a nanocrystal.

voids in the film is expected due to the $\mathrm{C}$ ions having a significant amount of energy when entering the amorphous carbon matrix.

A bright field image of a nanocrystalline region whose crystallites gives $d$-spacings close to those of cubic diamond is shown in Fig. 2(a). The number of nanocrystals that give a $d$-spacing close to that of diamond (under the electron beam) decreases with time due to charging. This is indicative that these crystals do not adhere well to the underlying ta-C film. The diffraction pattern from such a crystallite is shown in Fig. 2(b). The films/crystallites were too thick in general for high resolution electron microscopy. These crystals were observed only on films deposited at the highest ion energy of $148 \mathrm{eV}$. Clusters of isolated crystalline regions separated by large areas of amorphous regions are found in the films. There are two types of crystallites observed in the ta-C film. The majority of the observed crystallites are large (0.5-2 $\mu \mathrm{m})$ and graphitic in nature. Diffraction patterns observed for these larger crystalline regions seem to indicate that the graphite crystals are oriented with the 002 plane lying close to the normal to the $\mathrm{Si}$ substrate. ${ }^{18}$ The graphite crystals show a large $\pi^{*}$ contribution at $285 \mathrm{eV}$ in the EELS spec- 


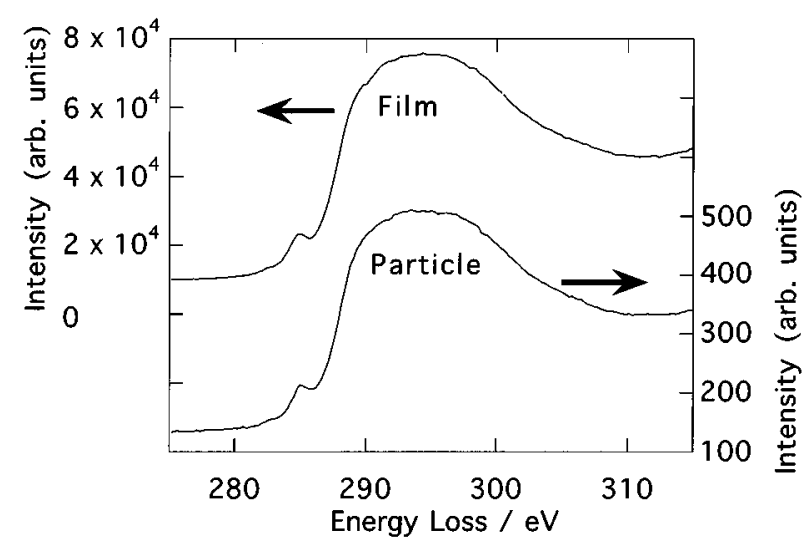

FIG. 3. The electron energy loss spectra of the $\mathrm{C} K$ edge at $\sim 285 \mathrm{eV}$ for the tetrahedral amorphous carbon film and from a nanocrystallite whose diffraction pattern is shown in Fig. 2.

trum. The nanocrystals that cannot be satisfactorily indexed to that of graphite give an EELS spectrum with a much smaller $\pi^{*}$ peak as shown in Fig. 3 .

The diffraction pattern obtained from the diamond nanoparticles in the ta-C film [Fig. 2(b)] have six diffraction rings visible, with the inverse ratio of the peak-to-peak diameters (which is proportional to $d_{h k l} / d_{111}$ ) following the sequence; $1.00,0.60 \pm 0.01,0.52 \pm 0.01,0.39 \pm 0.01,0.34 \pm 0.01$, and $0.29 \pm 0.006$. The observed sequence is close to that expected for cubic diamond; 1.00, 0.61, 0.52, (0.43), 0.40, 0.35, 0.33, $(0.31), 0.29, \ldots$ etc. with the diffraction rings corresponding to 0.43 and 0.31 not being present in the diffraction pattern obtained. The mean values of the $d$ spacing calculated using the camera length is shown in Table I. The observed $d$ spacing from the nanocrystals closely match those of cubic diamond.

The EELS data obtained from one of the diamond nanoparticles and that of the surrounding amorphous ta-C film is shown in Fig. 3. A wide energy range EELS scan (0-1200 $\mathrm{eV}$ ) of the particles and the ta-C film does not show edges that can be identified with impurities. Therefore, it is concluded that the diffraction pattern shown in Fig. 2(b) is not due to an impurity such as a metal that could give rise to similar $d$ spacings. Also the $L$ edge due to oxygen is not present in the samples indicating the ta-C films are of good quality. When an analysis similar to that of Berger et al. ${ }^{13}$ is performed on the $\mathrm{C} K$ edge EELS spectra, $s p^{2}$ contents of

TABLE I. Observed and published $d$ spacings for diamond.

\begin{tabular}{|c|c|c|c|c|}
\hline \multirow[b]{2}{*}{$(h k l)$} & \multicolumn{2}{|c|}{ ASTM Card No. 6-675 } & \multicolumn{2}{|c|}{ Experiment } \\
\hline & $\begin{array}{c}\text { Published } d \\
(\AA)\end{array}$ & Intensity & $\begin{array}{l}\text { Observed } d \\
(\AA)\end{array}$ & Intensity \\
\hline 111 & 2.06 & 100 & 2.08 & very strong \\
\hline 200 & 1.785 & forbidden & & \\
\hline 220 & 1.261 & 25 & 1.24 & strong \\
\hline 311 & 1.075 & 16 & 1.07 & medium \\
\hline 222 & 1.030 & forbidden & & \\
\hline 400 & 0.892 & 8 & & \\
\hline 331 & 0.818 & 16 & 0.815 & weak \\
\hline $422 / 333$ & $0.728 / 0.686$ & & 0.707 & weak \\
\hline 440 & 0.630 & & & \\
\hline 531 & 0.603 & & 0.605 & very weak \\
\hline
\end{tabular}

$12.7 \pm 5 \%$ and $14.8 \pm 5 \%$ are obtained for the background amorphous ta-C film and the observed diamond nanocrystallites, respectively. The EELS analysis indicates the chemical composition of the crystallites to be composed entirely of carbon, with a majority of its bonding structure being tetrahedral. Based on the fact that the chemical composition of the nanocrystallites is carbon, together with the diffraction data shown in Fig. 2(b), it is believed that diamond nanocrystallites are observed in the ta-C films under certain bias conditions.

In summary, nanocrystallites having a chemical composition of carbon and giving a diffraction pattern that is within $2 \%$ of the published values of cubic diamond are observed in room-temperature deposited tetrahedral amorphous carbon using the filtered cathodic vacuum arc. The nanocrystallites may have formed either within the arc in the plasma phase or due to the kinetic processes involved in the rapid thermal quenching of the $\mathrm{C}^{+} / \mathrm{C}^{++}$ions. It is possible that graphitic macroparticles are necessary for the preseeding of the diamond nanocrystals as the majority of the crystallites found in the films are graphitic in nature.

${ }^{1}$ D. R. McKenzie, D. Muller, and S. A. Pailthorpe, Phys. Rev. Lett. 67, 773 (1991).

${ }^{2}$ P. J. Fallon, V. S. Veerasamy, C. A. Davis, J. Robertson, G. Amaratunga, W. I. Milne, and J. Koskinen, Phys. Rev. B 48, 4777 (1993).

${ }^{3}$ S. R. P. Silva, K. Knowles, G. Amaratunga, and A. Putnis, Diam. Relat. Mater. 3, 1048 (1994).

${ }^{4}$ E. F. Chaikovski, V. Puzikov, and A. Semenov, Sov. Phys. Crystallogr. 26, 122 (1981).

${ }^{5}$ Y. Namba and T. Mori, J. Vac. Sci. Technol. A 3, 319 (1985).

${ }^{6}$ S. R. P. Silva, G. A. J. Amaratunga, E. K. H. Salje, and K. M. Knowles, J. Mater. Sci. 29, 4962 (1994).

${ }^{7}$ S. Anders, A. Anders, I. G. Brown, B. Wei, K. Komvopoulos, J. W. Ager, III, and K. M. Yu, Surf. Coat. Technol. 68/69, 388 (1994).

${ }^{8}$ R. Lossy, D. L. Pappas, R. A. Roy, J. J. Cuomo, and V. M. Sura, Appl. Phys. Lett. 54, 216 (1992).

${ }^{9}$ M. Weiler, S. Sattel, K. Jung, H. Ehrhardt, V. S. Veerasamy, and J. Robertson, Appl. Phys. Lett. 64, 2797 (1994).

${ }^{10}$ G. M. Pharr, D. L. Callahan, S. D. McAdams, T. Y. Tsui, S. Anders, A. Anders, J. W. Ager, I. G. Brown, C. Singh, S. R. P. Silva, and J. Robertson, Appl. Phys. Lett. 68, 779 (1996).

${ }^{11}$ Y. Yin, D. R. McKenzie, J. Zou, and A. Das, J. Appl. Phys. 79, 1563 (1996).

${ }^{12}$ S. Jou, H. J. Doerr, and R. F. Bunshah, Thin Solid Films 253, 95 (1994).

${ }^{13}$ S. D. Berger, D. R. McKenzie, and P. J. Martin, Philos. Mag. Lett. 57, 285 (1988).

${ }^{14}$ R. F. Egerton, Electron Energy Loss Spectroscopy in the Electron Microscope (Plenum, New York, 1986).

${ }^{15}$ D. R. McKenzie et al., Diam. Relat. Mater. 1, 773 (1995).

${ }^{16}$ Y. Lifshitz, G. D. Lempert, E. Grossman, I. Avigal, C. Uzan-Saguy, R. Kalish, J. Kulik, D. Marton, and J. W. Rabalais, 4, 318 (1995)

${ }^{17}$ S. Xu, B. K. Tay, H. S. Tan, S. R. P. Silva, and W. I. Milne, J. Appl. Phys. 79, 7234 (1996).

${ }^{18}$ S. R. P. Silva, S. Xu, B. K. Tay, H. S. Tan, H. J. Scheibe, M. Chhowalla, and W. I. Milne, Surf. Coat. Technol. (in press).

${ }^{19}$ M. Chhowalla, J. Robertson, S. R. P. Silva, and G. A. J. Amaratunga (unpublished).

${ }^{20}$ X. Oks et al., IEEE Trans. Plasma Sci. (in press).

${ }^{21}$ J. J. Cuomo, D. L. Pappas, J. Bruley, J. Doyle, and K. L. Saenger, J. Appl. Phys. 70, 1706 (1991). 